\title{
Frege and saving substitution
}

\author{
Bryan Pickel $^{1} \cdot$ Brian Rabern $^{2}$
}

Accepted: 5 October 2020/ Published online: 16 October 2020

(C) The Author(s) 2020

\begin{abstract}
Goodman and Lederman (Philos Stud 177(4):947-952, 2020) argue that the traditional Fregean strategy for preserving the validity of Leibniz's Law of substitution fails when confronted with apparent counterexamples involving proper names embedded under propositional attitude verbs. We argue, on the contrary, that the Fregean strategy succeeds and that Goodman and Lederman's argument misfires.
\end{abstract}

Keywords Leibniz' Law · Substitution · Frege - Sense - Ambiguity · Opacity · Validity

\section{Introduction}

On one formulation of Leibniz's Law, terms flanking true identities are everywhere intersubstitutable salva veritate: "given a true statement of identity, one of its two terms may be substituted for the other in any true statement and the result will be true" (Quine 1953: 139). This may be regimented as the following schema, where $\Phi[\alpha / \beta]$ is the result of substituting an occurrence of $\alpha$ for $\beta$ in $\Phi$.

$$
\text { Substitution: }(\alpha=\beta \wedge \Phi) \rightarrow \Phi[\alpha / \beta]
$$

Codifying this, it is tempting to hold that the schema substitution and its instances are valid.

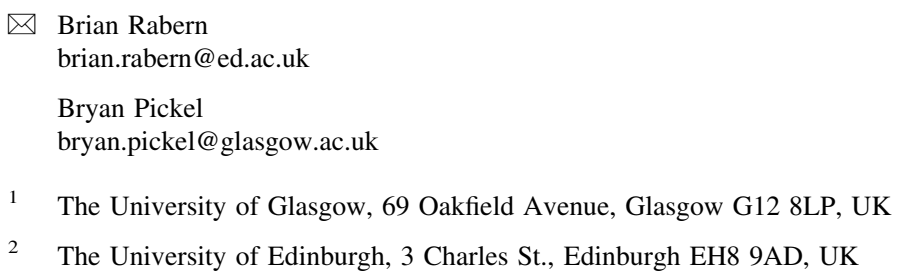


Yet, it has been argued that instances of suBstitution cannot be valid because they have false uses involving quotation, modal, and epistemic contexts. Focussing on epistemic contexts, any typical utterance of sentence (1) would likely be taken as false.

(1) If George Eliot is Mary Anne Evans and Twain knows that George Eliot wrote Middlemarch, then Twain knows that Mary Anne Evans wrote Middlemarch.

Although George Eliot is Mary Anne Evans, some who assert 'George Eliot wrote Middlemarch' would reject 'Mary Anne Evans wrote Middlemarch'. It is a genuine discovery that Mary Anne Evans wrote Middlemarch even to those who know that George Eliot did. This has led various theorists to insist that the truth of the antecedent of (1) does not require the truth of the consequent. Thus we have an apparently false reading of (1) and a counterexample to the validity of sUBSTITUTION.

According to the traditional Fregean diagnosis, these false uses of (1) do not threaten the validity of SUBSTITUTION. As (1) is normally used, the first occurrences of 'George Eliot' and 'Mary Anne Evans' refer to their customary referents, the woman herself, whereas the second occurrences (which occur under attitude verbs) refer to the senses of 'George Eliot' and 'Mary Anne Evans', respectively. The fact that the ambiguous sentence has a false reading is no more a counterexample to SUBSTITUTION, than is the false use of sentence (2) with the first three occurrences of 'Aristotle' referring to Aristotle Onassis, the famous shipping magnate, and the fourth referring to the philosopher:

(2) If Aristotle is Aristotle and Aristotle is a shipping magnate, then Aristotle is a shipping magnate.

One way to formally handle the sort of ambiguity posited by the Fregean-what Kaplan (1968) calls fanatical mono-denotationalism-would be to introduce distinct expressions for uses of names that have distinct referents. In fact, Frege himself recommended this method:

To avoid ambiguity, we ought really to have special signs in indirect speech, though their connection with the corresponding signs in direct speech should be easy to recognise.

(Frege's 28-12-1902 letter to Russell; translated in Frege 1902/1980, 153)

On this approach, (1) is not an instance of sUBSTITUTION at all on account of its ambiguity. For the purposes of assessing validity, ambiguous sentences should be replaced by their disambiguations. Thus, the false reading of (1) will be disambiguated as $\left(1^{*}\right)$, where an underlined expression refers to the customary sense of the expression without an underline. ${ }^{1}$

\footnotetext{
1 This follows Frege's suggestion in his letter to Russell dated 28-12-1902. Of course, Frege doesn't ever implement this convention (nor any other way of treating indirect contexts) into his official formalism, but in the letter to Russell he uses the device of underlining to distinguish customary and indirect reference (and double underlining for doubly indirect reference). Church's formalization of Frege explicitly adopts such a method, where he uses subscripts to disambiguate (see his Logic of Sense and
} 
(1*) If George Eliot is Mary Anne Evans and Twain knows that George Eliot wrote Middlemarch, then Twain knows that Mary Anne Evans wrote Middlemarch.

This sentence is not an instance of SUBSTITUTION and therefore its falsity is no threat to the validity of the law.

A more relaxed Fregean approach tolerates some ambiguity in assessing validity. ${ }^{2}$ There are various ways to construe validity when dealing with ambiguous languages. We will focus on one natural way to extend the notion of validity as applied to unambiguous languages. On this approach, a schema is valid only if all uniform disambiguations of its instances are true (or at least not false). ${ }^{3}$

The injunction not to equivocate in the course of an argument makes the ambiguities disappear for logical purposes. (Kaplan 1986, 262)

The falsity of (1) on most uses is due to the fact that its terms are not uniformly disambiguated. Uniform disambiguations of (1) can be represented by (1a) and (1b).

(1a) If George Eliot is Mary Anne Evans and Twain knows that George Eliot wrote Middlemarch, then Twain knows that Mary Anne Evans wrote Middlemarch.

(1b) If George Eliot is Mary Anne Evans and Twain knows that George Eliot wrote Middlemarch, then Twain knows that Mary Anne Evans wrote Middlemarch.

Disambiguation (1a) is true because its antecedent is false if 'George Eliot' and 'Mary Ann Evans' are taken to have their customary referent, namely the woman herself-the conjunct 'Twain knows that George Eliot wrote Middlemarch' is false. ${ }^{4}$ Disambiguation (1b) is true because 'George Eliot is Mary Anne Evans' says falsely

\section{Footnote 1 continued}

Denotation in Church 1951). See also Introduction to Mathematical Logic where Church speaks of "eliminating the oblique uses of names by introducing special names to denote the senses which other names express" (Church 1956: 8). See also Kaplan (1964) and Klement (2002: chapter 4).

2 Instead of analyzing the different contributions of an ambiguous expression in its different occurrences by introducing two expressions which univocally make these different contributions, an alternative strategy complicates the semantics for a single expression so that it makes different contributions in its different occurrences. See Pickel and Rabern (forthcoming) for discussion of these two methods for implementing Frege's proposal (cf. Kaplan 1964: 22-23).

3 See Lewis (1982: 438-441) for discussion of various options for a logic of ambiguity. Since Goodman and Lederman (2020) make their case in terms of the Kaplanian conception whereby there is an injunction against equivocation our diagnosis does as well.

4 According to Frege, Thoughts are the only objects of propositional attitudes. But 'Twain knows that George Eliot wrote Middlemarch' on the relevant disambiguation states that Twain stands in a relation to something that's not a Thought. "A truth value cannot be a part of a Thought, any more than, say, the Sun can, for it is not a sense but an object" (Frege 1892/1952: 64). This argument assumes that the sense of 'wrote Middlemarch' can compose with the referent of 'George Eliot' resulting in something that's not a Thought. One way to support this would be to assume that the sense of 'wrote Middlemarch' is a function (Church 1951; Geach 1976, and Pickel (unpublished)) and follow Frege in assuming that all functions are total. An alternative approach would say that (1a) is undefined on the relevant disambiguation, in which case it is at least not false. 
of the senses of the expressions 'George Eliot' and 'Mary Anne Evans' that they are the same. Thus, the fact that (1) is false on an equivocal use doesn't threaten the validity of SUBSTITUTION. In this way the Fregean can save the principle of SUBSTITUTION. ${ }^{5}$

\section{The argument and validity}

Goodman and Lederman (2020) argue that this Fregean strategy of explaining away apparent failures of SUBSTITUTION-by appeal to equivocation-leads to undesirable results, when combined with certain cherished principles of epistemic logic, such as what is known must be true. In their initial presentation, Goodman and Lederman offer an argument against the validity of (1) that rests on the validity of (3).

(3) If Kripke knows that George Eliot is Mary Anne Evans, then George Eliot is Mary Anne Evans

Goodman and Lederman's argument makes use of auxiliary premises. We will first consider and reject a straightforward version of this argument that makes use of auxiliary premises that the Fregean should accept. Given these auxiliary premises, we will argue that the Fregean has reason to reject the validity of (3). We will then consider and reject a more sophisticated version of the argument-closer to the text of Goodman and Lederman-that makes use of different auxiliary premises. In the case of this more sophisticated argument, we will argue that the Fregean should reject the auxiliary premises.

The straightforward version of the argument presupposes two principles that we will call UNIFORMITY and CLOSURE.

UNIFORMITY: Validities are true on every uniform disambiguation.

CLOSURE: Validity is closed under classical propositional logic.

We have already seen that the Fregean should endorse UNIFORMITY so as not to invalidate subSTITUTION. Classical propositional logic preserves truth on a uniform disambiguation. So if some sentences are true on a uniform disambiguation, their consequences will also be true on that uniform disambiguation.

The problem is that (4) follows from (1) and (3) by classic propositional logic.

(4) If Kripke knows that George Eliot is Mary Anne Evans and Twain knows that George Eliot wrote Middlemarch, then Twain knows that Mary Anne Evans wrote Middlemarch.

\footnotetext{
5 Note that the Fregean also applies this strategy to apparent counterexamples to suBstituTION stemming from quotation. Consider the following: "If Cicero is Tully and 'Cicero' contains six letters, then 'Tully' contains six letters." The Fregean will insist that the names in the sentence are used equivocally-the first occurrences of 'Cicero' and 'Tully' refer to their customary referents, the man himself, whereas the second occurrences refer to the names themselves 'Cicero' and 'Tully', respectively. An argument analogous to Goodman and Lederman's that used quotational contexts and a T-out principle, $\operatorname{Tr}(\ulcorner\Phi\urcorner) \rightarrow \Phi$, instead of attitude contexts and factivity, would misfire for the reasons analogous to the ones we give below. In this way, Frege doesn't even need to restrict substitution to non-quotational contexts, as many other theorist do (cf. Kaplan 1968, 185-186).
} 
Therefore, by Closure, (4) is valid as well. But (4) is false even on the uniform disambiguation that disambiguates all occurrences of 'George Eliot' and 'Mary Anne Evans' as 'George Eliot' and 'Mary Anne Evans', respectively. Thus, by UNIFORMITY, (4) is not valid. This contradicts the assumption that both (1) and (3) are valid. Because they take for granted at this point that (3) is valid, Goodman and Lederman would suggest rejecting the validity of (1), and more generally of Leibniz's Law of substitution. ${ }^{6}$

However, we believe that this rejects the wrong premise. Given UNIFORMITY - that every validity is true on every uniform disambiguation - it is clear that (3) cannot be valid. This might be surprising given the acceptability of normal utterances of (3). However, there is a difference between validity and acceptability for the Fregean. The Fregean should reject the validity of (3) despite the acceptability of its normal utterances, just they endorse the validity of (1) despite the unacceptability of its normal utterances. According to the Fregean, a normal utterance of (3) that is true would involve equivocation. The Fregean will disambiguate any usual utterance of (3) as the mixed disambiguation $\left(3^{*}\right)$, where the expressions under the attitude verb in the antecedent refer to their customary senses, but in the consequent they refer to their customary references.

If Kripke knows that George Eliot is Mary Anne Evans, then George Eliot is Mary Anne Evans.

This disambiguation corresponds to a normal utterance of (3) and it is true, but this doesn't show that (3) is valid. The requirement on validity outlined above was that any uniform disambiguation should yield a truth. But a uniform disambiguation of (3) would either interpret the names as referring to their customary references as in (3a) or their customary senses as in (3b) (or perhaps higher level senses).

(3a) If Kripke knows that George Eliot is Mary Anne Evans, then George Eliot is Mary Anne Evans.

(3b) If Kripke knows that George Eliot is Mary Anne Evans, then George Eliot is Mary Anne Evans.

Disambiguation (3a) is true. In particular, (3a) has a false antecedent since the occurrence of 'George Eliot is Mary Anne Evans' refers to a truth-value instead of a Thought. But crucially, (3b) is not true. The antecedent of (3b) is true, since it makes the ordinary claim that Kripke stands in the knowledge relation to the Thought usually expresses by 'George Eliot is Mary Anne Evans'. But the consequent of $(3 \mathrm{~b})$ refers to something that is not the True-namely the Thought that George Eliot is Mary Anne Evans. According to Frege's semantics, a material conditional refers to the False if the antecedent refers to the True while the consequent refers to something that is not the True (see Frege (1893/2013), §12). Thus (3b) is false, and therefore (3) has at least one uniform disambiguation that fails to be true. The argument here needn't rest on Frege's own semantics for the

\footnotetext{
${ }^{6}$ In footnote 2 Goodman and Lederman insist that their argument could rely on the sentence "If the thought that Hesperus is Phosphorus is a true thought, then Hesperus is Phosphorus" instead of sentence (3). Our response to this case would parallel our response to the present argument.
} 
material conditional. Most reasonable accounts will say that a conditional with a true antecedent and a consequent that is not true is itself not true. Thus, the conditional (3b) is not true. Therefore, by UNIFORMITY, (3) is not valid. ${ }^{7}$

The argument we've given so far subtly differs from the argument first considered by Goodman and Lederman (2020) in that they rely on a premise weaker than UNIFORMITY. Rather, they rely on a principle we will call UNIFORMITY* in addition to CLOSURE.

UNIFORMITY*: Validities are not false on any uniform disambiguation.

CLOSURE: Validity is closed under classical propositional logic.

The premise UNIFORMITY* does not require a validity to be true on every uniform disambiguation, but only that a validity fails to be false on every uniform disambiguation. Given our discussion above, one could reject Frege's own semantics for the conditional and hold that (3) is not false on disambiguation (3b) - instead it is undefined or gappy, and so merely untrue on this disambiguation. Thus, if UNIFORMITY* is assumed instead of UNIFORMITY, the untrue uniform disambiguation of (3) does not establish that it is invalid. ${ }^{8}$ Appealing to UNIFORMITY*, Goodman and Lederman can offer an argument against the validity of (1) with the same structure as before.

In order for this argument to work, it must be possible for a sentence to be valid while being untrue on some uniform disambiguations. In particular, the argument takes as a premise that (3) is valid according to the relevant notion of validity. But it is untrue under some uniform disambiguations. Thus, this version of the argument requires not merely the truth of UNIFORMITY*, but the falsity of UNIFORMITY. The problem, from our point of view, is that familiar notions of validity with this structure do not satisfy CLOSURE. In particular, classical propositional logic preserves truth at a disambiguation. ${ }^{9}$ We should not expect it to preserve lack of falsity at a

\footnotetext{
7 Our argument here makes a substantive assumption about the notion of disambiguations. In particular, we assume that any way of disambiguating the ambiguous sub-expressions of a sentence provides a disambiguation of the sentence. And thus we assume that (3a) and (3b) are uniform disambiguations of (3). One might resist this assumption and insist that an unembedded name can only be disambiguated as referring to its customary referent whereas a name embedded in a belief ascription can only be disambiguated as referring to its customary sense. On this approach, (3) has no uniform disambiguations because the two occurrence of 'George Eliot' and the two occurrences of 'Mary Anne Evans' are embedded under different numbers of attitude operators and so cannot be disambiguated in the same way, respectively. One might try to uphold the validity of (3) by maintaining that it has no uniform disambiguations at all, and hence no false ones. This is related to the notion of weak validity in Goodman and Lederman (2020). A schema is weakly valid when all of its embedding uniform instances are valid. A sentence $S$ is embedding uniform when every occurrence of an expression in $S$ is embedded under the same number of attitude verbs. If an expression is not embedding uniform, then it has no uniform disambiguations on the approach under consideration. As a result, it will not be false on any uniform disambiguations. Goodman and Lederman argue, and we agree, that weak validity is not a theoretically important status.

${ }^{8}$ Note that Goodman and Lederman don't highlight the difference between UNIFORMITY* and UNIFORMITY-we are doing so on their behalf. They don't actually consider the relevant uniform interpretation of (3) at all.

9 "There is indeed a sense in which classical logic preserves truth even in the presence of ambiguity. If an implication is classically valid, then for every unmixed disambiguation of the entire implication, in
} 
disambiguation. For instance, Goodman and Lederman (2020: footnote 2) themselves observe (following Smiley 1967) that the notion of Strawsonvalidity - truth in all contexts where a sentence's presuppositions are satisfied-is not closed under classical propositional logic. Strawson-validity is not closed under classical propositional logic because the conclusion of an argument may be false at a context where the premises are merely untrue due to their presuppositions being unsatisfied. Analogously, a uniform disambiguation of an argument could make the conclusion false while making the premises merely untrue.

As we have said, the argument presupposes that (3) is valid on the relevant notion of validity. Given that (4) follows from (1) and (3) and yet is false on some uniform disambiguations (and therefore invalid), the Fregean should take this as prima facie evidence that the relevant notion of validity is not closed under classical propositional logic. This is not to say that there can be no notion of "validity" that satisfies CLOSURE and allows for validities that are untrue at a uniform disambiguation. But the relevant notion of validity must then be shown to be relevant to the Fregean's projects, and we don't see such an attempt in Goodman and Lederman (2020).

\section{The argument and schmalidity}

We have examined the argument that Goodman and Lederman provisionally consider for the invalidity of SUBSTITUTION and argued that one of its premises-that (3) is valid-is false. However, Goodman and Lederman move to an argument that rests on a different premise, that sentence (3) typifies that "good status, however precisely it is understood...at which systematic theorizing about knowledge aims". They call this good status schmalidity. We'll argue that the Fregean has no more reason to be impressed by an argument that appeals to schmalidity than they do to an argument that appeals to validity.

To infer that (1) is invalid from the fact that (3) is schmalid, Goodman and Lederman offer principles governing schmalidity. Paralleling our discussion above, we first examine straightforward versions of these principles that might govern this notion. We show that the schmalidity of (3) is not compatible with these principles.

SUBSET: Validities are schmalidities.

UNIFORMITY-S: Schmalidities are true on every uniform disambiguation.

CLOSURE-S: Schmalidity is closed under classical propositional logic.

From the validity of (1), it follows that (1) is schmalid, by SUBSET. From the schmalidity of (1) and (3) and CLOSURE-s, it follows that (4) is schmalid. But from the fact that (4) has a false uniform disambiguation, it follows by UNIFORMITY-s that (4) is not schmalid. The argument is then taken as a reductio of the schmalidityand therefore validity-of (1).

Footnote 9 continued

which each ambiguous constituent is disambiguated the same way throughout all the premises and the conclusion, the conclusion is true on that disambiguation if the premises are." (Lewis 1982: 440) 
But given that (3) is schmalid, UNIFORMITY-S is not true. In particular, the argument above showed that (3) is not true under every uniform disambiguation. At best, it is true under any normal disambiguation. But these normal disambiguations are not uniform. Thus, the good status enjoyed by (3) is not of the sort that requires truth under every uniform disambiguation.

In their text, Goodman and Lederman appeal to a weaker version of UNIFORMITY-s. That is, they appeal to UNIFORMITY-S*.

UNIFORMITY-S*: Schmalidities are not false on any uniform disambiguation.

The principle UNIFORMITY-S* is compatible with the schmalidity of (3) even though (3) has an untrue uniform disambiguation. If (3) is schmalid, then it is possible for a sentence to be both schmalid and untrue on a uniform disambiguation. But, parallel to the discussion above, this brings CLOSURE-s into question. Schmalidity again has a structural similarity to Strawson-validity. Classical propositional logic preserves truth on a disambiguation. But we see no reason to expect it to preserve lack of falsity on a disambiguation. Therefore, the Fregean should be no more impressed by this argument than they were by the argument above.

\section{Fregean factivity}

But can the Fregean do justice to the apparent "good status" of (3)? We think so. As we mentioned, the Fregean will disambiguate any usual utterance of (3) as the mixed disambiguation $\left(3^{*}\right)$, which is true. This disambiguation isn't easily statable as an obviously valid schema, such as $\ulcorner\operatorname{Knows}(\Phi) \rightarrow \Phi\urcorner$, because the instances of $\Phi$ and the terms they contain will need to be disambiguated differently in the different contexts. However, the fact that ordinary assertions of (3) almost always are disambiguated as $\left(3^{*}\right)$ is - we believe - a sign of good status.

Goodman and Lederman might still ask: can the Fregean offer a valid principle that does justice to the factivity of knowledge? Fortunately, they can. The gloss of factivity mentioned above-what is known is true-can be straightforwardly regimented as a validity. Let us introduce an operator True(.), the referent of which applies to a Thought just in case that Thought presents the True.

FREGEAN FACTIVITY: $\operatorname{Knows}(\Phi) \rightarrow \operatorname{True}(\Phi)$

The Fregean can accept this as a valid schema. Notice that it is true on any uniform disambiguation: So long as $\Phi$ refers to a Thought, if someone stands in the knowledge relation to the referent of $\Phi$, then the referent of $\Phi$ presents the True.

What's more, this factivity schema has the following valid instance.

(5) If Kripke knows that George Eliot is Mary Anne Evans, then it is true that George Eliot is Mary Anne Evans.

This instance is interesting because Frege is a redundancy theorist of truth. The sense of an utterance of $\ulcorner$ It is true that $\Phi\urcorner$ is the same as the sense of a 
corresponding utterance of $\Phi .{ }^{10}$ This means that although the Fregean should deny that (3) is valid, there is a natural picture according to which ordinary utterances of (3) express the same Thought as a uniform disambiguation of a validity, namely (5). In this respect, ordinary utterances of (3) are in very good logical standing. ${ }^{11}$

Importantly, this good feature of (3) - ordinarily expressing the same Thought as a uniform disambiguation of a logical truth-is not preserved under logical consequence and doesn't require truth under all uniform disambiguations. Thus, if the feature we have characterised is called "schmalidity", then CLOSURE-S and UNIFORMITY-S are both false.

\section{Conclusion}

We have argued that the Fregean need not accept the classical consequences of whatever good status is enjoyed by sentence (3), because (3) is not true on all uniform disambiguations. This blocks Goodman and Lederman's argument for the invalidity of (1). However, the fact that (3) is not true on all uniform disambiguations does not entail that ordinary utterances of (3) are untrue just as holding that (1) is valid does not entail that ordinary utterances of (1) are true. Instead the Fregean ought to endorse the validity of (5), and more generally the schema FREGEAN FACTIVITY. In light of this, the Fregean can still maintain that (3) has very good logical status in that any ordinary utterance will be true and will express the same Thought as a valid sentence under a uniform disambiguation.

There is a methodological lesson here. The good (or bad) status of various utterances of sentences in ordinary reasoning does not automatically align with their validity. If a language exhibits ambiguities, then these ambiguities may ordinarily be resolved according to certain patterns. However, there is no antecedent reason to presuppose that these patterns correspond to the resolutions required to assess the validity of the ambiguous sentence. In the case of (1), although it is true on any uniform disambiguation, it is typically unacceptable. In contrast, although (3) is typically acceptable, it's false or untrue on some uniform disambiguations. Therefore, given the choice-adopted by Fregeans such as Kaplan-to assess validity in terms of uniform disambiguations, (1) is valid but unacceptable and (3) is invalid but acceptable. Acceptability of normal utterances should not be conflated with validity. ${ }^{12}$

\footnotetext{
10 "...[T]he sense of the word 'true' is such that it does not make any essential contribution to the thought. If I assert 'It is true that sea-water is salt', I assert the same thing as if I assert 'Sea-water is salt'. (Frege 1915/1997, 323). The precise of notion correspondence will need to be spelled out. An anonymous referee for THIS JOURNAL suggests roughly that an utterance of $\Phi$ and $\ulcorner$ it is true that $\Phi\urcorner$ correspond just in case the sense of each occurrence of an elementary expressions in the former is the referent of its occurrence in the latter.

${ }^{11}$ See Blanchette (2012) on Frege's conception of logic as concerned with Thoughts not syntactical items.

12 There is a suggestive connection here to the the view of Harman (1986) whereby there is a separation of the principles of validity from the principles of good reasoning. See also Stebbing (1930/1942, 473-5) and Burgess (2005).
} 
For a language that exhibits the sort of ambiguities posited by the Fregean various theoretical choices must be made about the operative notion of validitychoices must even be made about the ultimate bearers of validity. We have, for the most part, made our case in terms of Goodman and Lederman's basic presuppositions about logic. Others may well question these background presuppositions, and thereby provide a different diagnosis of Goodman and Lederman's argument. For example, one might question the assumption that the bearers of validity are ambiguous sentence types instead of disambiguated sentence types, or sentences in a context. (We are sympathetic.) A proponent of genuine opacity may make a different choice about how to assess validity in an ambiguous language. But there is still no antecedent guarantee that validity will entail ordinary acceptability or viceversa.

Acknowledgements For comments on earlier drafts many thanks to Wolfgang Schwarz, David Chalmers, James Shaw, and an anonymous referee.

Open Access This article is licensed under a Creative Commons Attribution 4.0 International License, which permits use, sharing, adaptation, distribution and reproduction in any medium or format, as long as you give appropriate credit to the original author(s) and the source, provide a link to the Creative Commons licence, and indicate if changes were made. The images or other third party material in this article are included in the article's Creative Commons licence, unless indicated otherwise in a credit line to the material. If material is not included in the article's Creative Commons licence and your intended use is not permitted by statutory regulation or exceeds the permitted use, you will need to obtain permission directly from the copyright holder. To view a copy of this licence, visit http:// creativecommons.org/licenses/by/4.0/.

\section{References}

Blanchette, P. A. (2012). Frege's conception of logic. Oxford: Oxford University Press.

Burgess, J. P. (2005). No requirement of relevance. In S. Shapiro (Ed.), Oxford handbook of philosophy of mathematics and logic (pp. 727-750). Oxford: Oxford University Press.

Church, A. (1951). A formulation of the logic of sense and denotation. In P. Henle, H. Kallen, \& S. Langer (Eds.), Structure, method and meaning: Essays in honor of H.M. Sheffer. New York: Liberal Arts Press.

Church, A. (1956). Introduction to mathematical logic (Vol. 13). Princeton: Princeton University Press.

Frege, G. (1892/1952). On Sense and Reference. In P. Geach and M. Black (Eds.) Translations from the philosophical writings of Gottlob Frege. New York: Philosophical Library.

Frege, G. (1893/2013). Gottlob Frege: basic laws of arithmetic. Oxford University Press.

Frege, G. (1902/1980). Frege to Russell (28-12-1902). In G. Gariel, H. Hermes, F. Kambartel, C. Thiel, A. Veraart, B. McGuiness \& H. Kaal (Eds.) Gottlob Frege: Philosophical and mathematical correspondence. Oxford: Basil Blackwell.

Frege, G. (1915/1997). My basic logical insights. In M. Beaney (Ed.), The Frege Reader (pp. 322-324). Blackwell Publishing.

Geach, P. T. (1976). Critical notice on Frege: Philosophy of language. Mind, 85, 436-449.

Goodman, J., \& Lederman, H. (2020). Sense, reference and substitution. Philosophical Studies, 177(4), 947-952.

Harman, G. (1986). Change in view: Principles of reasoning. Cambridge: The MIT Press.

Kaplan, D. (1964). Foundations of intensional logic, PhD thesis, UCLA.

Kaplan, D. (1968). Quantifying in Synthese, 19(1/2), 178-214.

Kaplan, D. (1986). Opacity, in L. E. Hahn and P. A. Schilpp (eds), The Philosophy of W. V. Quine, Open Court, pp. 229-289. 
Klement, K. C. (2002). Frege and the logic of sense and reference. New York: Routledge.

Lewis, D. (1982). Logic for equivocators. Nô̂s, 16(3), 431-441.

Pickel, B. (unpublished). The functional composition of sense.

Pickel, B., \& Rabern, B. (forthcoming). The myth of occurrence-based semantics, Linguistics and Philosophy pp. 1-25.

Quine, W. V. (1953). Reference and modality. In From a logical point of view (pp. 139-159). Cambridge: Harvard University Press.

Smiley, T. (1967). Mr. Strawson on the traditional logic. Mind, 76(301), 118-120.

Stebbing, L. S. (1930/1942). A modern introduction to logic. London: Methuen \& Co. LTD.

Publisher's Note Springer Nature remains neutral with regard to jurisdictional claims in published maps and institutional affiliations. 